\title{
Video Article \\ Microfluidic Picoliter Bioreactor for Microbial Single-cell Analysis: Fabrication, System Setup, and Operation
}

\author{
Alexander Gruenberger ${ }^{1}$, Christopher Probst ${ }^{1}$, Antonia Heyer ${ }^{1}$, Wolfgang Wiechert ${ }^{1}$, Julia Frunzke ${ }^{1}$, Dietrich Kohlheyer $^{1}$ \\ ${ }^{1}$ Institute of Bio- and Geosciences, IBG-1: Biotechnology, Forschungszentrum Juelich GmbH
}

Correspondence to: Dietrich Kohlheyer at d.kohlheyer@fz-juelich.de

URL: https://www.jove.com/video/50560

DOI: doi: $10.3791 / 50560$

Keywords: Bioengineering, Issue 82, Soft lithography, SU-8 lithography, Picoliter bioreactor, Single-cell analysis, Polydimethylsiloxane, Corynebacterium glutamicum, Escherichia coli, Microfluidics, Lab-on-a-chip

Date Published: 12/6/2013

Citation: Gruenberger, A., Probst, C., Heyer, A., Wiechert, W., Frunzke, J., Kohlheyer, D. Microfluidic Picoliter Bioreactor for Microbial Single-cell Analysis: Fabrication, System Setup, and Operation. J. Vis. Exp. (82), e50560, doi:10.3791/50560 (2013).

\section{Abstract}

In this protocol the fabrication, experimental setup and basic operation of the recently introduced microfluidic picoliter bioreactor (PLBR) is described in detail. The PLBR can be utilized for the analysis of single bacteria and microcolonies to investigate biotechnological and microbiological related questions concerning, e.g. cell growth, morphology, stress response, and metabolite or protein production on single-cell level. The device features continuous media flow enabling constant environmental conditions for perturbation studies, but in addition allows fast medium changes as well as oscillating conditions to mimic any desired environmental situation. To fabricate the single use devices, a silicon wafer containing sub micrometer sized SU-8 structures served as the replication mold for rapid polydimethylsiloxane casting. Chips were cut, assembled, connected, and set up onto a high resolution and fully automated microscope suited for time-lapse imaging, a powerful tool for spatio-temporal cell analysis. Here, the biotechnological platform organism Corynebacterium glutamicum was seeded into the PLBR and cell growth and intracellular fluorescence were followed over several hours unraveling time dependent population heterogeneity on single-cell level, not possible with conventional analysis methods such as flow cytometry. Besides insights into device fabrication, furthermore, the preparation of the preculture, loading, trapping of bacteria, and the PLBR cultivation of single cells and colonies is demonstrated. These devices will add a new dimension in microbiological research to analyze time dependent phenomena of single bacteria under tight environmental control. Due to the simple and relatively short fabrication process the technology can be easily adapted at any microfluidics lab and simply tailored towards specific needs.

\section{Video Link}

The video component of this article can be found at https://www.jove.com/video/50560/

\section{Introduction}

Time-lapse microscopy is a powerful tool for studying living cells in vivo ${ }^{1}$. Meanwhile commercially available fully automated microscopy platforms including thermally induced focus drift compensation are commonly applied in biological research to study time-dependent phenomena, ranging from cancer and neuron cell research over tissue engineering and dynamic studies with single yeast or bacterial cells ${ }^{2-6}$

Typically transparent well plates, agar-pads, or simply microscopy slides are applied to provide cell culture environments during time-lapse imaging ${ }^{7}$. Even though suitable for certain research, these simple systems have very limited control over environmental conditions and do not allow for more complex perturbations or well defined and fast medium changes. Disposable microfluidic chip devices produced by mass production have been introduced to the market recently but are mostly tailored towards larger eukaryotic cell types ${ }^{4}$. Although growth can be followed, well defined growth investigations concerning, e.g. precise cell trapping, colony size, growth direction, and the ability for cell removal are limited. Microfluidic habitats and reactors, in which bacteria cells are cultured in 3D environments have been developed ${ }^{8-10}$, have drawbacks when dealing with quantitative studies at the single-cell level. While overall population heterogeneity can be analyzed, many cell parameters cannot be determined accurately with single-cell resolution since growth is not restricted to monolayers.

This limitation triggered the development of microsystems enabling the cultivation of cells in well-defined channels and habitats with cells growing in flat monolayers with single cell resolution and especially tight control over media supply and environmental conditions ${ }^{6,11,12}$. Few examples of microfluidic systems for the cultivation of bacterial cells have been demonstrated ${ }^{12-14}$. Bacteria typically exhibit very fast growth rates and require microfluidic structures in the range of few micrometers and below, especially when cell monolayers are desired for microscopy. Keymer et al. demonstrated growth and spreading of $E$. coli strains in microfabricated landscapes ${ }^{15,16}$. Since they were interested in population dynamics they did not investigate with single-cell resolution.

We have developed the picoliter bioreactor (PLBR $)^{13}$, which is currently applied to investigate various biotechnological performance indicators such as growth ${ }^{17}$ and fluorescence coupled productivity analysis on single-cell level ${ }^{18,19}$. The present microfluidic device allows environmental reactor control at a defined culture volume of approximately one picoliter and continuous single-cell observation simultaneously. In comparison to open monolayer box systems ${ }^{11,14}$, where one or two sides are open to the media supply channel, the PLBR allows for controlled trapping 
and culturing. The design permits long term cultivation of bacteria without the risk of several adjacent colonies forming one large population. Furthermore, the system incorporates cultivation regions of $1 \mu \mathrm{m}$ height (in the order of the cell diameter) to restrict bacteria growth to cell monolayers. In contrast, the supply channels are 10 fold deeper to minimize hydraulic resistance.

In comparison to miniaturized batch cultivation systems ${ }^{20}$ the present system allows the cultivation with constant environmental parameters due to continuous media flow. Furthermore, environmental parameters such as medium composition, temperature, flow rates and gas exchange can be easily controlled and changed within seconds. This allows for specific investigations of cellular response to environmental changes concerning for example nutrient availability or stress stimuli. The demand for reduced media volumes, namely in the range of few microliters only, enable researchers to perform novel studies, e.g. the perturbation of cells during time-lapse imaging with supernatant of large-scale experiments unraveling cell response under these specific environmental conditions ${ }^{17}$. The picoliter bioreactor provides researchers with a robust system that tightly controls biophysical conditions and is operated using high precision syringe pumps and automated bright field and fluorescence microscopy for time-lapse imaging. Here, we report a complete protocol including device design, fabrication, and exemplary applications.

\section{Wafer Fabrication}

1. Design the microfluidic device containing inlets, outlets, main channels and the PLBRs (Figure 1A) using CAD software.

2. The design presented in this protocol (Figure 2) consists of two seeding inlets, a gradient generator for mixing of two different substrates, one outlet, and six arrays of PLBRs. Each array contains 5 PLBRs, resulting in 30 parallel PLBRs inside one microfluidic device.

3. Create a lithography photomask containing the desired chip layouts (Figure 1B). The photomask was produced in-house by electron beam writing with submicron resolution. The mask used was composed of a chromium layer on a 5 in $^{2}$ glass plate.

Note: perform all following steps under cleanroom class 100 conditions or better (a process flowchart is shown in Figures $3 \mathrm{~A}$ and 3B).

4. Clean a 4 in silicon wafer with piranha (10:1 ratio of sulfuric acid and hydrogen peroxide) and hydrofluoric acid for several minutes (Caution: hazardous chemicals). Rinse with deionized (DI) water for approximately $10 \mathrm{sec}$.

5. Dehydrate wafer for $20 \mathrm{~min}$ at $200^{\circ} \mathrm{C}$.

6. Spin coat $1 \mu \mathrm{m} \mathrm{SU}-82000.5$ photoresist onto the wafer ( $1^{\text {st }}$ layer $)(4 \mathrm{ml}$ resist, spin $10 \mathrm{sec}$ with, $v=500 \mathrm{rpm}$, and a $=100 \mathrm{rpm} / \mathrm{sec}, \mathrm{spin} 30$ $\mathrm{sec}$ with $\mathrm{v}=1,000 \mathrm{rpm}$ and $\mathrm{a}=300 \mathrm{rpm} / \mathrm{sec}$ ).

7. Place the coated wafer on a hotplate at $95^{\circ} \mathrm{C}$ to drive off excess solvent $\left(1.5 \mathrm{~min}\right.$ at $65^{\circ} \mathrm{C}, 1.5 \mathrm{~min}$ at $95^{\circ} \mathrm{C}$, and $1 \mathrm{~min}$ at $65^{\circ} \mathrm{C}$; ideally use two hotplates).

8. Insert $1^{\text {st }}$ layer photomask (here the trapping regions of the picoliter reactors) and wafer inside the mask aligner and expose wafer to $350-400$ $\mathrm{nm}$ (vacuum contact, $64 \mathrm{~mJ} / \mathrm{cm}^{2}, \mathrm{t}=3 \mathrm{sec}, \mathrm{l}=7 \mathrm{~mW} / \mathrm{cm}^{2}$ ).

9. Perform post exposure bake on a hotplate at $95^{\circ} \mathrm{C}$ to initiate the polymerization of SU-8 $\left(1 \mathrm{~min}\right.$ at $65^{\circ} \mathrm{C}, 1 \mathrm{~min}$ at $95^{\circ} \mathrm{C}$, and $1 \mathrm{~min}$ at $\left.65^{\circ} \mathrm{C}\right)$. Note: after this step the structures in the SU-8 layer can be seen.

10. Place the wafer in a SU-8 developer bath for 1 min and transfer the wafer into a second container with fresh SU-8 developer for few seconds.

11. Rinse the wafer in isopropanol to remove SU-8 developer and dry wafer using nitrogen flow of wafer spinner.

12. Hard bake the wafer for $10 \mathrm{~min}$ at $150^{\circ} \mathrm{C}$.

13. Spin coat $9 \mu \mathrm{m}$ SU-8 2010 photoresist onto the wafer $\left(2^{\text {nd }}\right.$ layer) (dispense $4 \mathrm{ml}$ resist, spin $10 \mathrm{sec}$ with $\mathrm{v}=500 \mathrm{rpm}$, a = $100 \mathrm{rpm} / \mathrm{sec}$, and spin $30 \mathrm{~s}$ with $v=4,000 \mathrm{rpm}, \mathrm{a}=300 \mathrm{rpm} / \mathrm{sec}$ ).

14. Place the wafer with SU-8 on a hotplate at $95^{\circ} \mathrm{C}$ to drive off excess solvent $\left(15 \mathrm{~min}\right.$ at $65^{\circ} \mathrm{C}, 45-60 \mathrm{~min}$ at $95^{\circ} \mathrm{C}$, and 10 min at $\left.65{ }^{\circ} \mathrm{C}\right)$. Note: attention has to be paid to wrinkles and bubbles. If the wafer is heated to fast to $95^{\circ} \mathrm{C}$, evaporated solvent may be encapsulated in tiny gas bubbles.

15. Insert photomask with the desired layout (here main channels for nutrient supply) and the wafer into the mask aligner and expose to $350-400$ $\mathrm{nm}$ (hard contact, $64 \mathrm{~mJ} / \mathrm{cm}^{2}, \mathrm{t}=10 \mathrm{sec}, \mathrm{l}=7 \mathrm{~mW} / \mathrm{cm}^{2}$ )

16. Perform post exposure bake on a hotplate at $95^{\circ} \mathrm{C}$ to finalize the polymerization of SU-8 $\left(5 \min\right.$ at $65^{\circ} \mathrm{C}, 3: 30 \mathrm{~min}$ at $95^{\circ} \mathrm{C}, 3 \mathrm{~min}$ at $\left.65{ }^{\circ} \mathrm{C}\right)$. Note: after this step the structures in the SU-8 layer can be seen.

17. Place the wafer in a SU-8 developer bath for $45 \mathrm{sec}$, transfer the wafer into a second container with fresh SU-8 developer and develop for 60 sec.

18. Rinse the wafer $20 \mathrm{sec}$ with isopropanol to remove any SU-8 developer residue and dry wafer using pressured nitrogen.

19. Finally hard bake the wafer at $150^{\circ} \mathrm{C}$. As a result the final wafer (Figure 1C) is obtained, which will be used as master mold for PDMS molding.

20. Perform profilometer measurements (Figure $3 \mathrm{C}$ ) to validate SU-8 structure heights. Note: inaccuracies in structure height may results in inefficient cell trapping or loss of cells during cultivation.

\section{Polydimethylsiloxane Chip Fabrication}

Note: All following steps should be ideally performed under laminar-flow conditions to prevent dust particles interfering with the fabrication procedure (A process flowchart is shown in Figure 4).

1. Prepare a mixture of polydimethylsiloxane (PDMS) base and curing agent in a 10:1 ratio. Mix carefully until a homogenous solution is achieved which looks opaque. Prepare as much as required for the desired layer height (here $3 \mathrm{~mm}$ ).

2. Degas the PDMS mixture for approximately 30 min under slight vacuum until all bubbles have disappeared.

3. Prepare molding device (or Petri dish) with appropriate SU-8 wafer and pour the PDMS mixture into it (Figure 1D).

4. Bake the PDMS for $3 \mathrm{hr}$ at $80^{\circ} \mathrm{C}$ in the oven.

5. Carefully peel off the PDMS slab from the wafer. Cut the slab into single chips using a clean and sharp scalpel. 
6. Wash the chips in a n-pentane bath for $90 \mathrm{~min}$, followed by two acetone washing baths (90 min each). Dry the chips overnight to remove any solvent residue. Caution: perform the PDMS washing under a fume hood. Note: during the $n$-pentane wash, monomers and dimers are removed from the cured PDMS and the chip size may temporarily double during washing procedure.

7. Store the microfluidic PDMS chips in close containers until the final experiment.

8. Just before the experiment, punch the inlet and outlet holes into the PDMS chip using a needle (or hole-puncher) with a slightly smaller diameter than the connectors that are used to connect tubing with PDMS chip.

9. Clean the microfluidic PDMS chip carefully with isopropanol and use scotch tape to remove any dust particles which might stick on the structured PDMS side. Use the scotch tape several times until no particle can be seen on the chip.

10. Clean a $170 \mu \mathrm{m}$ thin glass slide with acetone and isopropanol successively. Finally clean with deionized water and dry with pressurized nitrogen.

11. Before plasma-activation, warm up the plasma cleaner and run the plasma for approximately 300 sec. Plasma-oxidize glass slide and PDMS chip (Power $50 \mathrm{~W}$, Time $=25 \mathrm{sec}$, oxygen flow rate $=20 \mathrm{sccm}$ ).

12. Align the PDMS and glass chip before bonding. Finally, place the PDMS chip carefully onto the glass slide (Figure 1E). PDMS and glass will bond within seconds. Note: do not push with tweezers onto the top of the PDMS chip during the bonding process. This may lead to so called roof-collapsing of the channels and small structures.

13. In order to strengthen the bond, bake the final PDMS-glass chip for $10 \mathrm{sec}$ at $80^{\circ} \mathrm{C}$.

\section{Preparation of the Bacterial Culture}

Note: All cultivations should be prepared in sterile filtered medium to prevent accumulation of undesired particles, which may interfere during cultivation.

1. Use an agar plate containing the desired organisms (here, C. glutamicum ATCC 13032) and inoculate one colony into $20 \mathrm{ml}$ of fresh BHI medium, incubate overnight $\left(\approx 8-14 \mathrm{hr}\right.$ ) at $30^{\circ} \mathrm{C}$ on a rotary shaker $(120 \mathrm{rpm})$.

2. Transfer $10 \mu \mathrm{l}$ of the preculture into the desired medium (here CGXII ${ }^{21}$ ) which will be used during microfluidic cultivation and let the cell grow overnight at $30^{\circ} \mathrm{C}$ on a rotary shaker (120 rpm).

3. Transfer the desired amount of cell suspension (between 10-500 $\mu \mathrm{l}$, depending on the start of the experiment) into the desired medium (here CGXII ${ }^{21}$ ) which will be used during microfluidic cell cultivation. Note: the best is to use cells from the early logarithmic phase for seeding. For $C$. glutamicum culture the best optical density $\left(O D_{600}\right)$ for seeding was between 0.5-2.

4. Transfer $1 \mathrm{ml}$ of the bacterial culture into a sterile $2 \mathrm{ml}$ tube. Note: this should be done right after the microfluidic PDMS chip was assembled to minimize transfer time between shake flask and microfluidic cultivation. Typically the transfer time is around 15 min and should be kept as small as possible to prevent impact on metabolism caused by oxygen limitation and temperature changes.

\section{Experimental Setup}

Note: All steps are performed with an inverted microscope.

1. Start microscope incubator 2 hrs before the experiment to warm-up the system. Note: the microscopy should be equipped with a fullsize incubator to control temperature and if desired gas flow. Additional humidity control is not necessary since the chip system is continuously infused with media.

2. Open incubator system, select the desired objective and if required add immersion oil onto the objective.

3. Mount the chip inside the chip holder. If required fix the glass plate with adhesive tape in order to avoid chip any movement during stage operation.

4. Center the sample on the microscope and focus onto the PLBR arrays.

5. Connect inlets and outlets with appropriate tubing (Figure 1F). Connect tubing to a waste reservoir. A representative chip can be seen in Figure 4D.

6. Insert syringes into pumps and start media flow. Use medium, buffer or if necessary coating solution and rinse the microfluidic channels for approximately $1 \mathrm{hr}$. Note: coating solution is used to coat channel walls to prevent unspecific cell adhesion.

7. For E. coli, $0.1 \%$ solution of BSA is used to coat the channel walls. For $C$. glutamicum no coating is necessary. After the coating procedure flush the chip with medium prior cell seeding.

8. Before cell seeding and cultivation, check that no leakage occurs and that the temperature is constant.

\section{Seeding of Bacterial Cells into the Microfluidic Device}

1. Make sure the desired bacteria solutions are available in appropriate syringes connected to tubing.

2. Disconnect buffer or coating solution and connect the cell suspension to the chip. To minimize death volume, undesired air bubbles and to reduce experimental time, change the complete needle as well as tubing, rather than only the syringes.

3. Infuse the cell suspension into the channels at a volumetric flow rate of $200 \mathrm{nl} / \mathrm{min}$ until most of the PLBRs are filled with the desired amount of cells (Figure 5A). Note: optimal seeding results depend on the bacterial strain, $\mathrm{OD}_{600}$, and growth medium of the preculture. These parameters have to be adapted to increase trapping efficiency and time until a sufficient number of cells are trapped in the reactor structures. For $C$. glutamicum, typically a cell suspension of $O D_{600} 0.5-2$ was used; for $E$. coli the $O D_{600}$ was between $0.5-1$.

4. If only a small number of PLBRs are filled, increase the flow rate to $800-1,200 \mathrm{nl} / \mathrm{min}$.

5. Disconnect the cell suspension and connect the growth medium to the chip (Figure 5B). Make sure that no air bubble is introduced during the medium change. Perfuse with fresh growth media at $100 \mathrm{nl} / \mathrm{min}$. 


\section{Time-lapse Imaging}

1. Select specific PLBRs for time-lapse imaging. Typically PLBRs are chosen that contain a single mother cell at the beginning of an experiment. The number of regions of interest that can be investigated in one experiment depends on the desired frame rate and microscopic setup.

2. Select an appropriate frame rate depending of number of PLBRs. Make sure that the microscope can handle the desired amount of ROls in the time lapse interval.

3. Choose appropriate filter sets (here YFP). Automatically close the shutter during stage movement and after each time-lapse measurement to prevent chromophore bleaching.

4. Configure the time-lapse microscopy sequence and start the experiment

5. After all PLBRs are overgrown, the experiment can be stopped, the microfluidic PDMS chip can be discarded and the experiment can be evaluated.

\section{Analysis}

Note: The following steps or parts of the procedure can be performed manually or by image analysis programs such as ImageJ, etc.

1. Determine PLBRs of interest where the cultivation fulfills all desired criteria, e.g. number of mother cells, position of the mother cells, etc.

2. To determine the growth rate of one microcolony count the number of cells in each time frame.

3. Calculate the maximum growth rate by plotting time vs. In (cell number). The slope of the plot represents the growth rate in [1/hr] (see Figure 6).

4. Fluorescence data analysis strongly depends on the performed experiment. In this report, an example was chosen to illustrate colony-tocolony and cell-to-cell heterogeneity between different isogenic microcolonies (see Figure 7).

\section{Representative Results}

\section{Device Fabrication}

The microfluidic PLBR system is fabricated by one layer of PDMS bonded onto a thin glass chip suited for high resolution microscopy. The fabrication consists of two main steps: firstly the fabrication of the replication master (Figures 1A, 1B, and 1C) and secondly the chip fabrication (Figures 1D, 1E, and 1F). According to the protocol, standard photolithographic microfabrication techniques are used to create the master mold. Laboratories without clean room facility can acquire commercially available customized SU-8 master molds. Using repetitive PDMS molding (Figures 1A, 1B, and 1C) hundreds of disposable chips can be produced. PDMS molding and chip assembly can be done in any lab and do not require clean room facilities, however, laminar airflow workplaces are favorable.

The process starts with the design of the microfluidic chip system. Typically CAD software is used to design the microfluidic chip (Figure 1A) After CAD, a mask is generated by an e-beam writer (Figure 1B) with submicron resolution. In the present study a 5 in chromium mask was created which was used for the SU-8 wafer lithography. The final silicon-SU-8 wafer is used for PDMS molding (Figure 1D). After a baking step the PDMS slab is cut into chips which are irreversibly bonded onto the glass slides (Figure 1E). Finally the tubing is connected (Figure 1F).

Figure 2 shows the design of the microfluidic system in detail. It consists of two seeding inlets, a gradient generator for mixing of different substrates or media and one outlet. The main channels have a dimension of $50 \mu \mathrm{m} \times 10 \mu \mathrm{m}(\mathrm{W} \times \mathrm{H})$. Each device consists of six arrays of PLBRs, containing 5 PLBRs each. This results in 30 parallelized reactors inside one microfluidic device.

Figure 3 illustrates the replication master production. As described in detail in the protocol, a first SU-8 layer is fabricated by SU-8 lithography (Figure 3A). A similar procedure is applied for the second layer (Figure 3B). To check the channel geometry we investigated the height of the PLBRs and main channels using a profilometer. In the example shown in Figure 3C, the first layer (the cultivation layer) was measured. Here the layer shows a consistent height of $1,200 \mathrm{~nm}$, suitable for the cultivation of C. glutamicum in BHI medium.

Figure 4 illustrates the PDMS molding procedure starting with PDMS mixing (Figure 4A) followed by the molding process (Figure 4B) and finally the bonding step (Figure 4C). Figure 4D shows the final microfluidic chip incorporating the $170 \mu \mathrm{m}$ thick glass plate, PDMS chip ( $3 \mathrm{~mm}$ in height) with inlets and outlets and steel needles connected to tubing. After the experiment the chip can be disposed and no extensive cleaning is necessary. Furthermore, it is easy to assemble and handle. No complex and difficult filling procedure is necessary.

\section{Device Principle}

Figure $\mathbf{5}$ shows the working principle of the reactor system. Cells are infused into the microfluidic device and individual cells remain trapped inside the PLBR simply by cell-wall interactions. Due to the difference in hydrodynamic resistance of channel and PLBR, only minimal flow occurs inside the PLBR. After seeding of the PLBR (Figure 5A), the growth and observation phase is initiated with a change from bacteria solution to growth medium (Figure $\mathbf{5 B}$ ). After the PLBRs are overgrown (Figure $\mathbf{5 C}$ ) the experiment is typically stopped and time-lapse images can be analyzed. For the trapping mechanisms and flow profile within the PLBR the reader is referred to Grünberger et al. ${ }^{13}$ for more details.

\section{Growth Rate Analysis}

The present system can be applied to study various bacterial species with respect to different biological parameters such as growth, morphology, or a fluorescent signal. In a first example C. glutamicum, an industrially relevant production organism was cultured under standard cultivation conditions $\left(\mathrm{T}=30^{\circ} \mathrm{C}, \mathrm{CGXII}\right.$ medium $\left.{ }^{21}\right)$. Figure $6 \mathrm{~A}$ shows the growth curves derived from three isogenic microcolonies. Exponential growth is 
maintained until the PLBRs are filled indicating that no nutrient limitation occurs. Figure 6B displays four DIC time-lapse microscopy images of a growing C. glutamicum colony.

\section{Fluorescence Analysis}

For single-cell fluorescence microscopy, researchers often make use of specific fluorescent proteins, for example GFP or derivatives, to couple a specific phenotype of interest to a measurable output (a fluorescent signal). To demonstrate the applicability of the PBLR for fluorescence based time-lapse studies, we investigated the fluorescence emission of a $C$. glutamicum strain producing a plasmid-encoded YFP-TetR fusion protein under control of the Ptac promoter (pEKEx2-yfp-tetR) ${ }^{18,22}$. In the presence of low inducer (IPTG) concentrations, expression from Ptac is known to lead to significant cell-to-cell variation in isogenic bacterial populations. Starting from one preculture, the growth and single cell fluorescence was followed for several isogenic microcolonies. As it can be seen in Figure 7, we observed phenotypic heterogeneity between different microcolonies and heterogeneity at the single-cell level within colonies starting from one mother cell. One colony (Figure 7B, PLBR 1) showed almost no fluorescence emission, whereas cells of PLBR 2 exhibited a low fluorescence emission due to basal yfp-tetR expression from the Ptac promoter. In PLBR 3 fluorescence emission was considerable strong compared to the other colonies and a broad distribution of the population was observed. This example demonstrates the applicability of the PBLR for time-lapse fluorescence microscopy studies. In comparison to flow cytometry, in which the fluorescence of single cells can be determined at one time point, the present systems allows the tracking of cells and the study of single-cell fluorescence in real time over many generations.

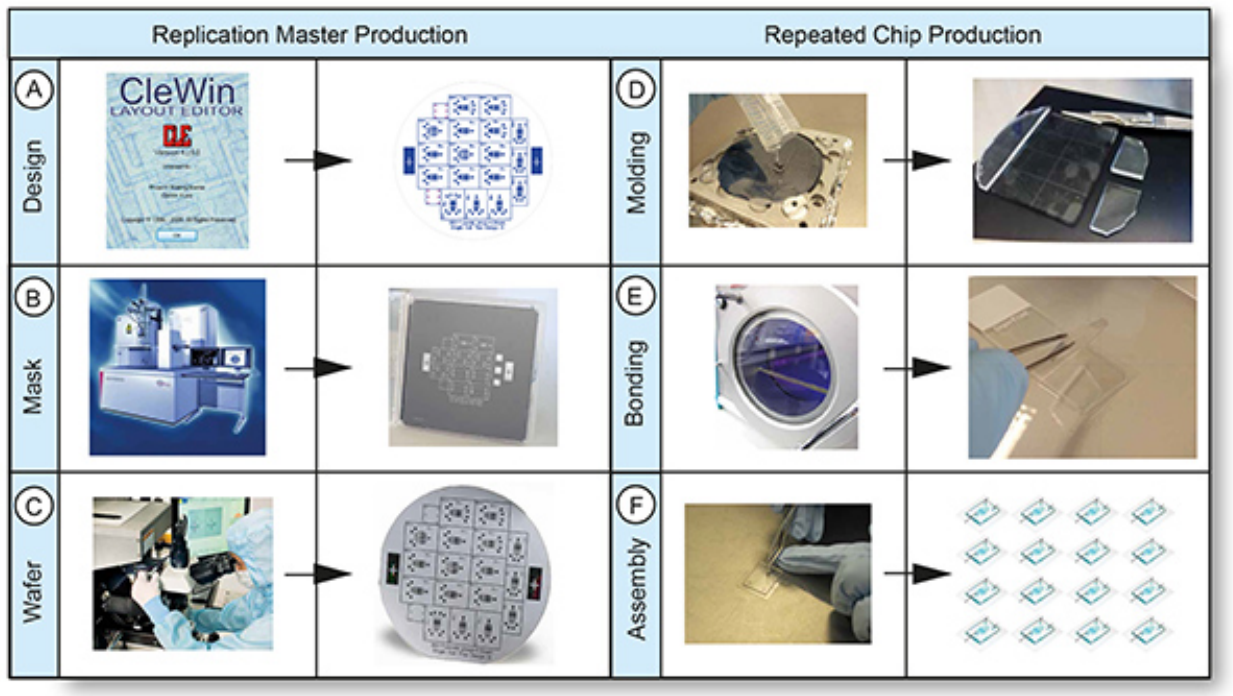

Figure 1. Overview of PLBR chip production process. Master mold fabrication: starting with (A) Design, (B) Lithography mask fabrication, and (C) Wafer production. PDMS-glass chip production: starting with (D) of PDMS molding followed by (E) glass and PDMS bonding and (F) final chip assembly. 

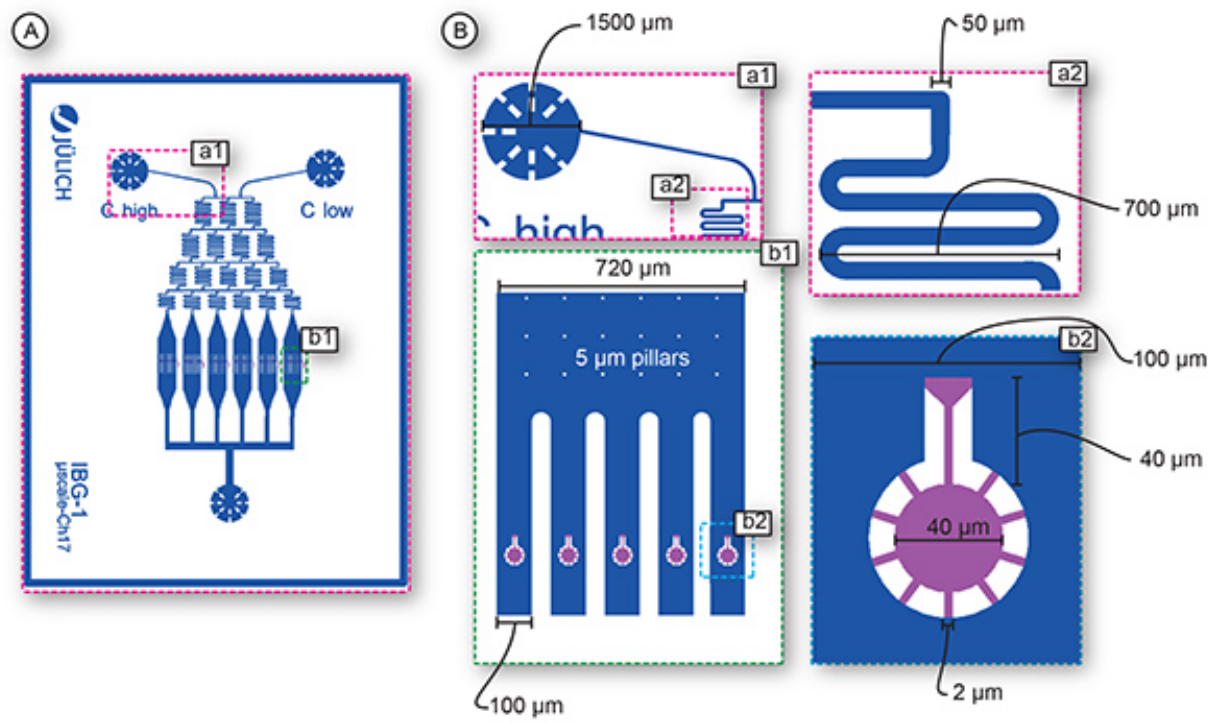

Figure 2. Design of the PLBR chip. (A) CAD drawing of the whole microfluidic chip; (B) Magnification of selected layout positions: The layout contains two medium inlets (a1), a gradient generator with mixing channels (a2) and 6 parallel PLBR arrays (b1). b2 shows one PLBR, which is embedded in a fluid channel with a width of $100 \mu \mathrm{m}$. The PLBR has an inner diameter of $40 \mu \mathrm{m}$ and nutrient channels with $2 \mu \mathrm{m}$ in width. The seeding inlet has a length of $40 \mu \mathrm{m}$. Pink color represents the first layer (trapping and cultivation region) and blue color represents the second layer (fluid transport).

(A)

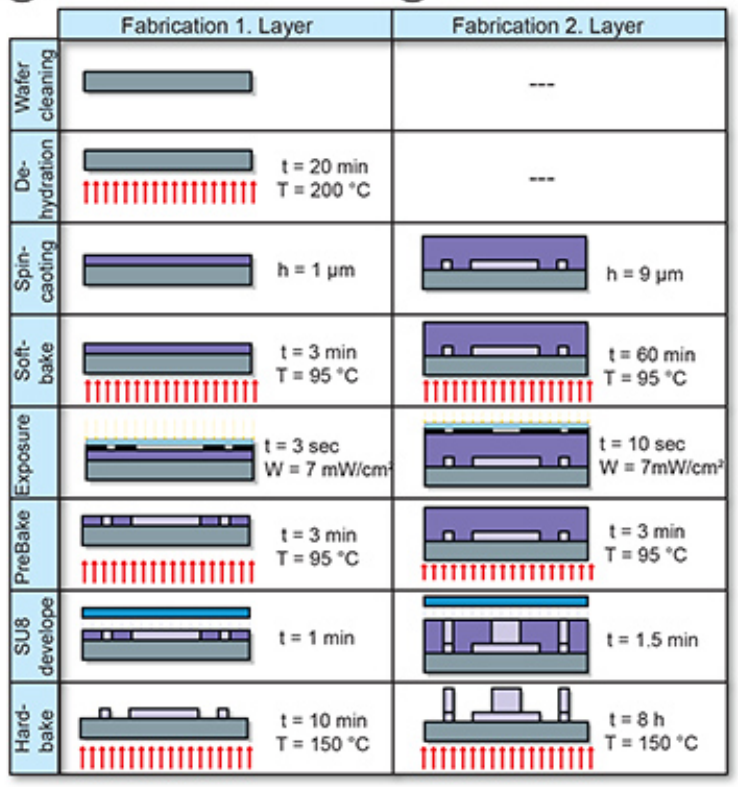

(C)

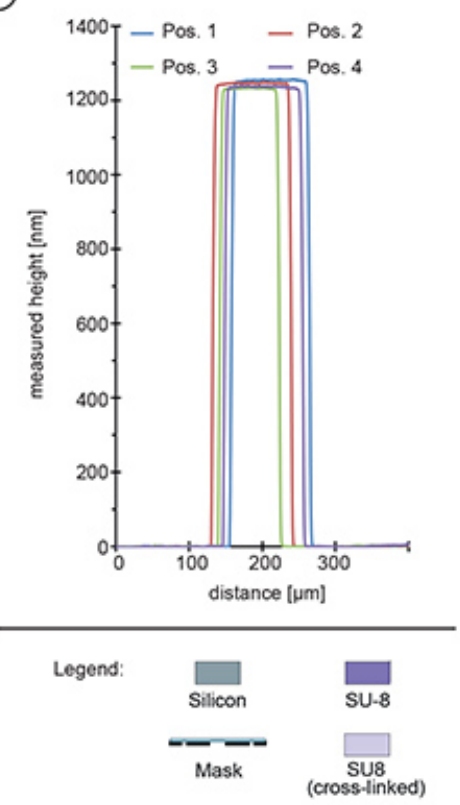

Figure 3. Illustration of two layer wafer fabrication process. (A) Fabrication of the first layer containing trapping structures; (B) Fabrication of the second layer containing fluid channels, inlets and outlets; (C) Representative surface profiles of the first layer. In this case the height of the first layer was $1,200 \mathrm{~nm}$ and is used for the cultivation of C. glutamicum in complex medium. 


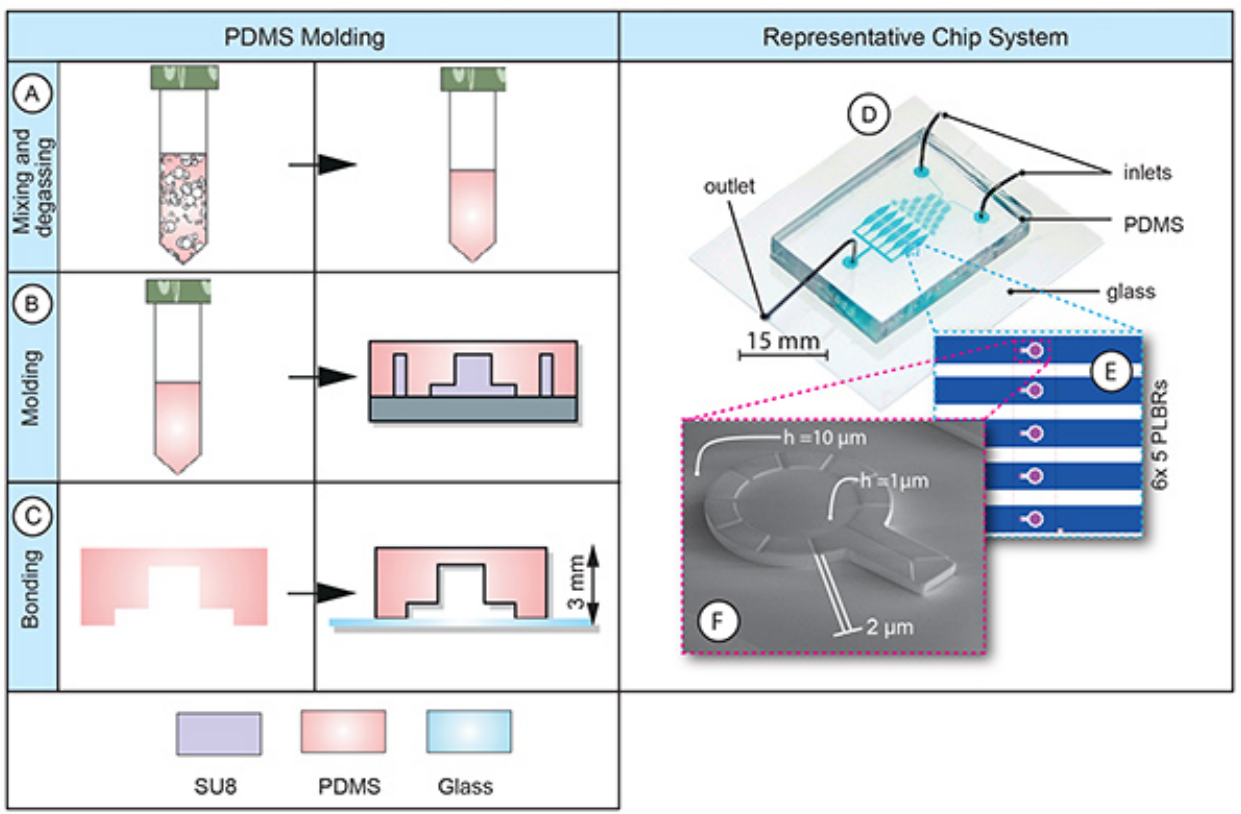

Figure 4. Device fabrication and representative chip. Illustration of the PDMS molding process: (A) PDMS mixing and degassing; (B) PDMS molding; (C) mold release, cutting and chip bonding. Final chip (Reproduced with permission of the Royal Society of Chemistry ${ }^{13}$ ): (D) photograph of the PDMS chip with 2 inlets and 1 outlet; (E) CAD image of six parallel arrays containing 5 PLBRs each; (F) SEM image of one PLBR

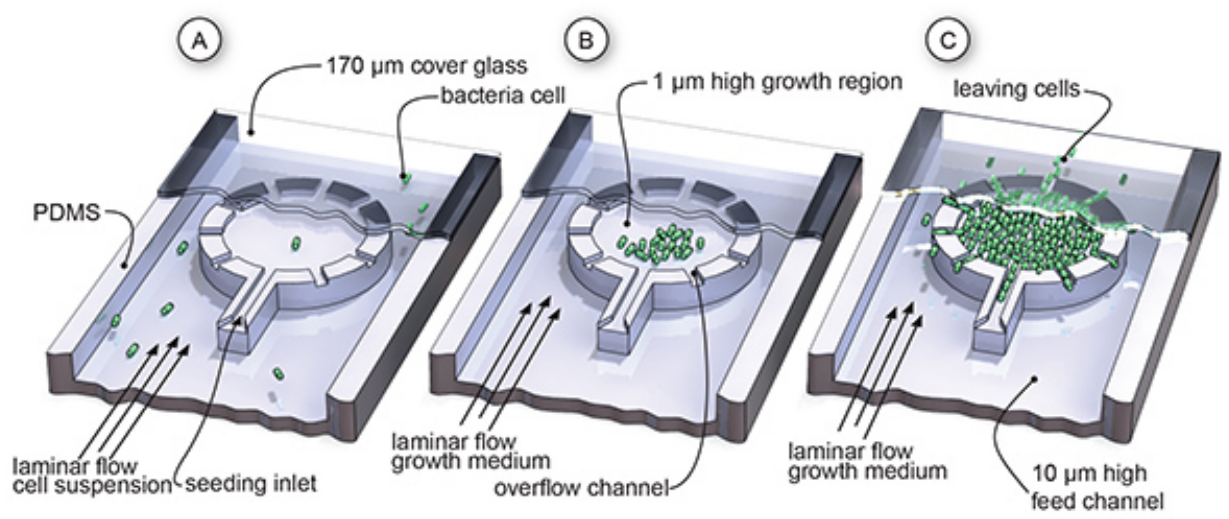

Figure 5. Working principle of the PLBR system. (A) Seeding phase; (B) Growth phase of the bacterial microcolonies; (C) Overflow phase. Reproduced with permission of the Royal Society of Chemistry13. http://dx.doi.org/10.1039/C2LC40156H. 
(A)

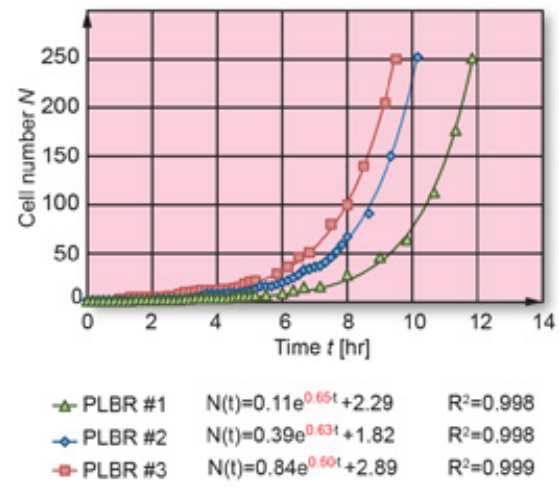

$$
\mu_{\max }\left[h^{\prime}\right]=0.63 \pm 0.02
$$

(B)
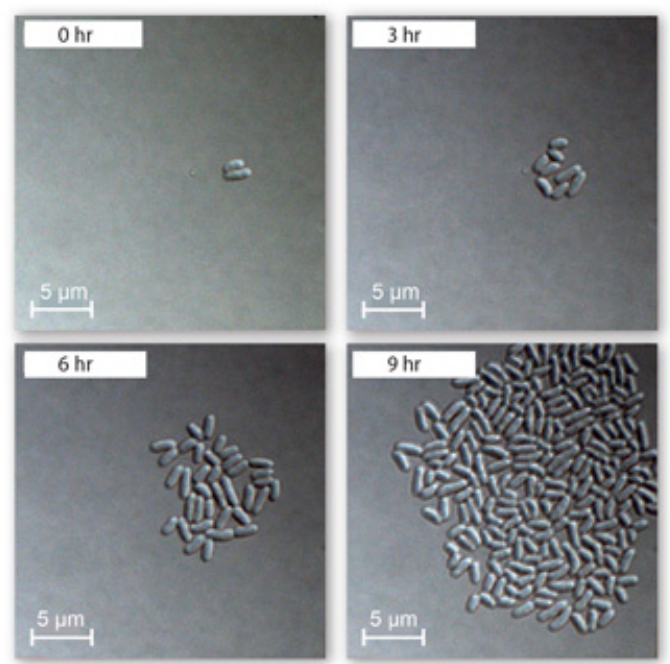

Figure 6. Growth rate determination of C. glutamicum WT microcolonies. (A) Growth plot of three PLBR cultivations and resulting exponential curves (Parts reproduced with permission of the Royal Society of Chemistry)13. http://dx.doi.org/10.1039/C2LC40156H. (B) Timelapse images of a growing C. glutamicum colony.

(A)

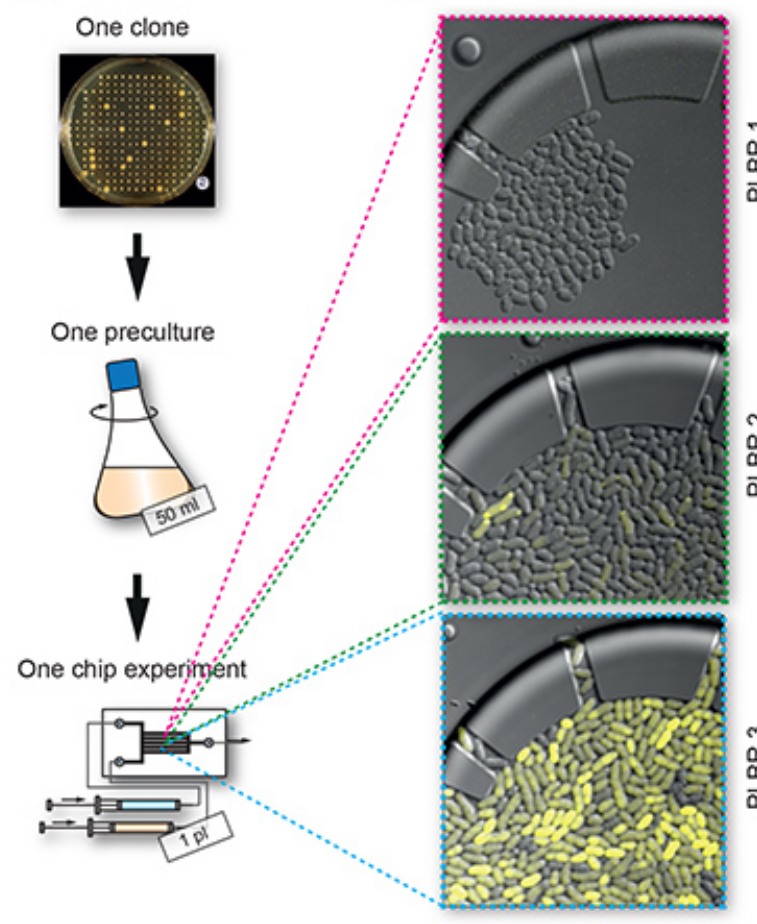

(C)
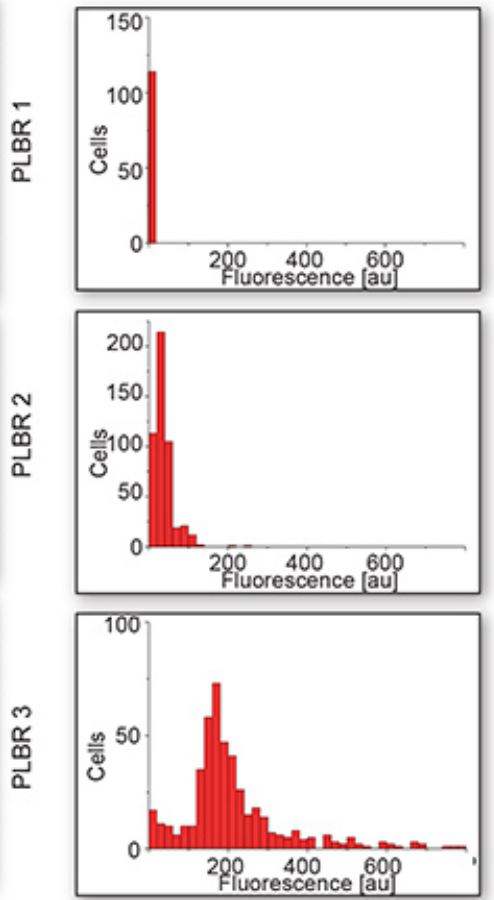

Figure 7. PBLR-based analysis of population heterogeneity. Shown is C. glutamicum expressing an yfp-tetR fusion under the control of the Ptac promoter (pEKEx2-yfp-tetR) in the absence of the inducer IPTG. (A) Experimental workflow; (B) Three isogenic microcolonies showing colony-to-colony heterogeneity and cell-to-cell heterogeneity; (C) Distribution of single-cell fluorescence within the respective microcolonies. 


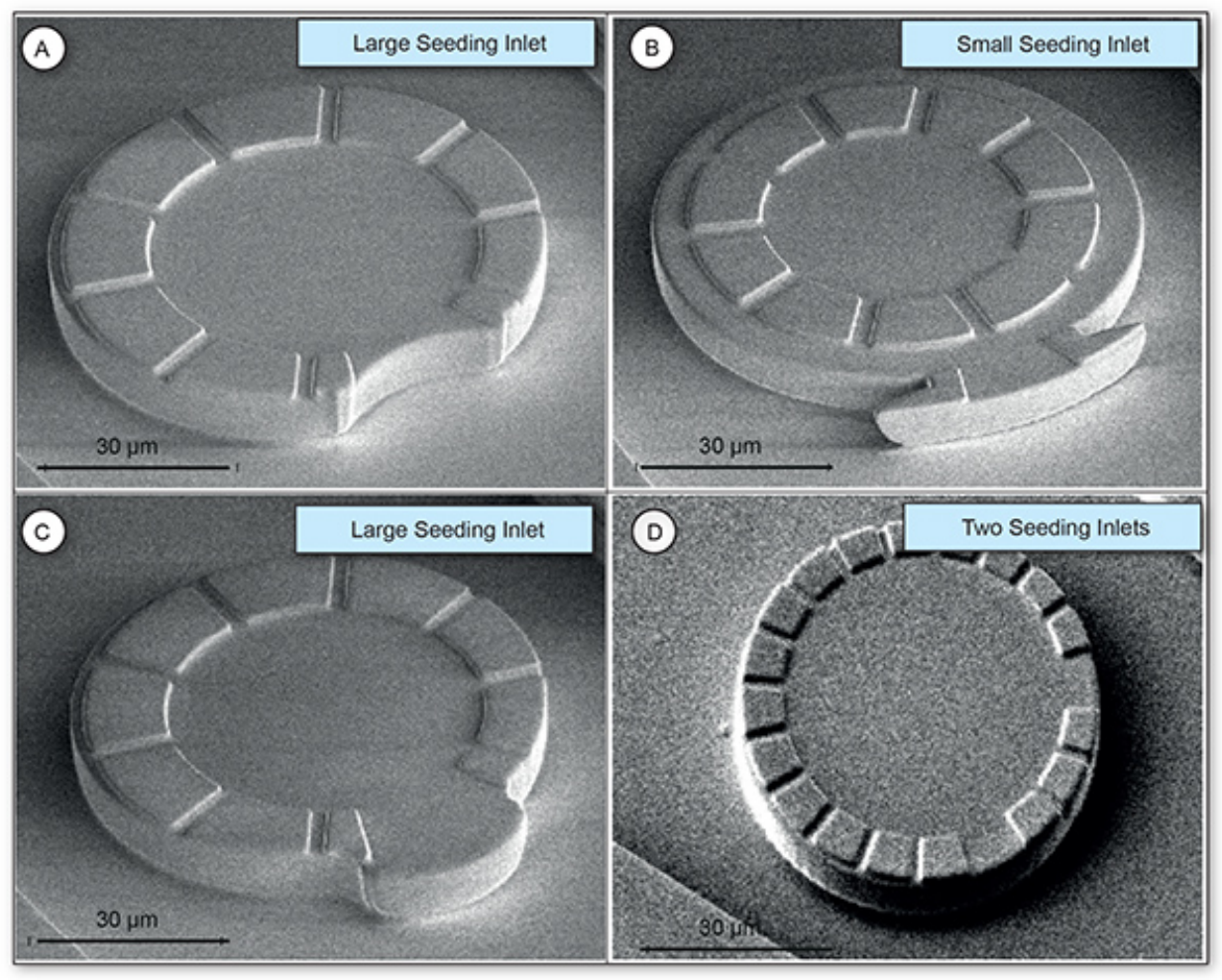

Figure 8. Scanning electron images of different PLBRs. SEM images showing seeding inlets for the optimization of trapping efficiency. (A) Lager seeding inlets (B) Smaller seeding inlets (C) Larger "open" seeding inlets (D) Two seeding inlets.

\begin{tabular}{|c|c|c|c|}
\hline Step & Problem & Possible Reason & Solution \\
\hline Wafer Fabrication & $\begin{array}{l}\text { Trapped air bubbles in SU-8 during } \\
\text { soft bake }\end{array}$ & Increase of temperature to fast & $\begin{array}{l}\text { Bake at } 95^{\circ} \mathrm{C} \text { and } 65^{\circ} \mathrm{C} \text { several } \\
\text { times }\end{array}$ \\
\hline Wafer Fabrication & $\begin{array}{l}\text { Disappearing and broken SU-8 } \\
\text { structures }\end{array}$ & $\begin{array}{l}\text { Not optimal fabrication procedure; } \\
\text { mechanical stress in SU-8 } \\
\text { structures }\end{array}$ & $\begin{array}{l}\text { Optimize parameter such as } \\
\text { baking time, exposure time }\end{array}$ \\
\hline Wafer Fabrication & $\begin{array}{l}\text { SU-8 layers to low or high or } \\
\text { uneven layer thickness }\end{array}$ & Problem during spin coating & $\begin{array}{l}\text { Check spin-coater parameters and } \\
\text { wafer chuck }\end{array}$ \\
\hline Chip Bonding and Assembly & Collapsing PLBRs & $\begin{array}{l}\text { PDMS bonding parameters not } \\
\text { optimal }\end{array}$ & $\begin{array}{l}\text { Adjust power, plasma exposure } \\
\text { time. and baking time after bonding }\end{array}$ \\
\hline Chip Bonding and Assembly & $\begin{array}{l}\text { Dirty structures and particles in the } \\
\text { PLBRs }\end{array}$ & Chip was not properly cleaned & $\begin{array}{l}\text { Apply scotch-tape for surface } \\
\text { cleaning }\end{array}$ \\
\hline Chip Bonding and Assembly & Insufficient PDMS-glass bonding & $\begin{array}{l}\text { Bonding parameters not optimal or } \\
\text { insufficient cleaning }\end{array}$ & Check settings of oxygen plasma \\
\hline Microfluidic Experiment & Fluid leakage & $\begin{array}{l}\text { Inlet/outlet hole was not properly } \\
\text { punched }\end{array}$ & Optimize hole punching process \\
\hline Microfluidic Experiment & $\begin{array}{l}\text { Many small PDMS particles during } \\
\text { filling }\end{array}$ & Hole was not properly punched & Optimize hole punching process \\
\hline $\begin{array}{l}\text { Microfluidic Experiment, Biological } \\
\text { Aspect }\end{array}$ & No cell growth & $\begin{array}{l}\text { Solvent residue from cleaning } \\
\text { procedure }\end{array}$ & $\begin{array}{l}\text { Flush chip more extensively prior } \\
\text { cell loading or let solvent evaporate } \\
\text { prior bonding }\end{array}$ \\
\hline $\begin{array}{l}\text { Microfluidic Experiment, Biological } \\
\text { Aspect }\end{array}$ & Changing growth rates & Various reasons & Check preculture and temperature \\
\hline $\begin{array}{l}\text { Microfluidic Experiment, Biological } \\
\text { Aspect }\end{array}$ & $\begin{array}{l}\text { Cell morphology changes during } \\
\text { cultivation }\end{array}$ & $\begin{array}{l}\text { Nutrient limitations or temperature } \\
\text { shift }\end{array}$ & Check incubator and flow \\
\hline $\begin{array}{l}\text { Microfluidic Experiment, Technical } \\
\text { Aspect }\end{array}$ & $\begin{array}{l}\text { Drift in position during time lapse } \\
\text { microscopy }\end{array}$ & Temperature fluctuations & $\begin{array}{l}\text { Check temperature profile prior } \\
\text { experiments until no oscillation }\end{array}$ \\
\hline $\begin{array}{l}\text { Microfluidic Experiment, Technical } \\
\text { Aspect }\end{array}$ & Loss of cells during cultivation & Slightly to high reactor height & Optimize reactor height \\
\hline
\end{tabular}




\begin{tabular}{|l|l|l|l|}
\hline $\begin{array}{l}\text { Microfluidic Experiment, Technical } \\
\text { Aspect }\end{array}$ & No trapping & Too low reactor height & Optimize reactor height \\
\hline
\end{tabular}

Table 1. Troubleshooting. This table summarizes critical aspects, common mistakes, and possible solutions during experimental work.

\section{Discussion}

We have described the fabrication, experimental setup and related operation procedures of a microfluidic PDMS device containing several (PLBRs) for single-cell analysis of bacteria.

Microfabrication using soft lithography techniques allows fast adjustments of device dimensions for various sizes and bacteria morphologies. Currently we are optimizing the picoliter bioreactor regarding the cultivation of different microbial organisms and cultivation throughput. In order to increase the trapping efficiency, also the reactor geometry is under optimization. Figure $\mathbf{8}$ shows four new PLBR devices that are currently validated. In all figures the seeding channel was redesigned with regards to width and shape. In practice, this seems to have an effect on the number of cells that are trapped, but needs further investigations. Significant improvements regarding the trapping efficiency were also achieved by the incorporation of additional overflow channels leading to higher convectional flow through the reactor and more cells being trapped. However, at the same time one increases the risk to wash out cells during cultivation.

The device is an interesting alternative to macroscale cultivations that have been used for decades to investigate growth and production processes on single-cell level. However, it has some important requirements: For parallel monitoring of several picoliter bioreactors a high resolution and fully motorized microscopic setup with focus drift compensation is mandatory. In addition an incubation system is needed to maintain the desired cultivation temperature constant throughout the measurements.

We achieve a $95 \%$ success rate in device fabrication. Main problems are related to inefficient PDMS-glass bonding, PDMS roof collapse, or fluid leakage (see Table 1 for troubleshooting of most occurring problems). Although the experimental work is done partially under nonsterile conditions, we rarely see contamination during experiments, due the closed fluidic system. PDMS microfluidic devices are optically transparent, therefore, can be used for high resolution in vivo imaging. Although PDMS seems to be perfect for the application, it has a high affinity for hydrophobic molecules, making the use of solvents which are widely used in whole cell biocatalytic processes limited. However, suitable coatings are available to adapt the protocol to these kinds of applications.

The proposed PLBR is well suited for spatio-temporal analysis of cellular and even subcellular events of various kinds of bacteria. A major advantage of the present approach lies in the ability to quantify microcolony growth directly in contrast to conventional methods. Furthermore, the PLBR allows for culturing under defined and constant conditions. Because the system facilitates the use of small amounts of reagents or materials it carries the advantages of being inexpensive, customizable and amenable to high throughput. In traditional methods average values of the whole population are considered when analyzing microbial cultivation. Furthermore, existing methods need manual sampling which can lead to degradation of samples and thus to errors in the measurement. The PLBR offers new perspectives for bioprocess development and population heterogeneity analysis in microbiology. The PLBR is a promising tool for various applications within bioprocess development and could be applied in various fields of research, e.g. analysis of cell-to-cell heterogeneity, analysis of specific cell clusters within cell-lineages, screening of microbial production strains and real-time investigation of cell phenotypes.

\section{Disclosures}

The authors have nothing to disclose.

\section{Acknowledgements}

The authors would like to thank the technician Agnes Müller-Schröer for her valuable contribution. This work was partly performed at Helmholtz Nanoelectronic Facility (HNF) at Forschungszentrum Jülich $\mathrm{GmbH}$. The authors are grateful for the generous help and support.

\section{References}

1. Muzzey, D. \& van Oudenaarden, A. Quantitative Time-Lapse Fluorescence Microscopy in Single Cells. Ann. Rev. Cell Dev. Biol. 25, 301-327, doi:10.1146/annurev.cellbio.042308.113408 (2009).

2. Wei, P. et al. Bacterial virulence proteins as tools to rewire kinase pathways in yeast and immune cells. Nature. 488, 384-388, doi:10.1038/ Nature11259 (2012).

3. Park, S. et al. Influence of topology on bacterial social interaction. Proc. Natl. Acad. Sci. U.S.A. 100, 13910-13915, doi:10.1073/ pnas.1935975100 (2003).

4. Lee, P. J., Helman, N. C., Lim, W. A. \& Hung, P. J. A microfluidic system for dynamic yeast cell imaging. BioTechniques. 44, 91-95, doi:10.2144/000112673 (2008).

5. Kobel, S. A. et al. Automated analysis of single stem cells in microfluidic traps. Lab Chip. 12, 2843-2849, doi:10.1039/C2LC40317J (2012).

6. Carlo, D. D., Wu, L. Y. \& Lee, L. P. Dynamic single cell culture array. Lab Chip. 6, 1445-1449, doi:10.1039/B605937F (2006).

7. Locke, J. C. W., Young, J. W., Fontes, M., Jimenez, M. J. H. \& Elowitz, M. B. Stochastic Pulse Regulation in Bacterial Stress Response. Science. 334, 366-369, doi:10.1126/science.1208144 (2011).

8. Boedicker, J. Q., Li, L., Kline, T. R. \& Ismagilov, R. F. Detecting bacteria and determining their susceptibility to antibiotics by stochastic confinement in nanoliter droplets using plug-based microfluidics. Lab Chip. 8, 1265-1272, doi:10.1039/B804911d (2008).

9. Fritzsch, F. S. O. et al. Picoliter nDEP traps enable time-resolved contactless single bacterial cell analysis in controlled microenvironments. Lab Chip. 13, 397-408, doi:10.1039/C2lc41092c (2013). 
10. Groisman, A. et al. A microfluidic chemostat for experiments with bacterial and yeast cells. Nat. Methods. 2, 685-689, doi:10.1038/Nmeth784 (2005).

11. Mather, W., Mondragon-Palomino, O., Danino, T., Hasty, J. \& Tsimring, L. S. Streaming Instability in Growing Cell Populations. Phys. Rev. Lett. 104, doi:10.1103/Physrevlett.104.208101 (2010).

12. Wang, P. et al. Robust growth of Escherichia coli. Curr. Biol. 20, 1099-1103, doi:10.1016/j.cub.2010.04.045 (2010).

13. Grunberger, A. et al. A disposable picolitre bioreactor for cultivation and investigation of industrially relevant bacteria on the single cell level. Lab Chip. 12, 2060-2068, doi:10.1039/c2lc40156h (2012).

14. Walden, M. \& Elf, J. Studying transcriptional interactions in single cells at sufficient resolution. Curr. Opin. Biotech. 22, 81-86, doi:10.1016/ j.copbio.2010.10.004 (2011).

15. Keymer, J. E., Galajda, P., Lambert, G., Liao, D. \& Austin, R. H. Computation of mutual fitness by competing bacteria. Proc. Natl. Acad. Sci. U.S.A. 105, 20269-20273, doi:10.1073/pnas.0810792105 (2008).

16. Keymer, J. E., Galajda, P., Muldoon, C., Park, S. \& Austin, R. H. Bacterial metapopulations in nanofabricated landscapes. Proc. Natl. Acad. Sci. U.S.A. 103, 17290-17295, doi:10.1073/pnas.0607971103 (2006).

17. Grunberger, A. et al. Beyond growth rate 0.6: Corynebacterium glutamicum cultivated in highly diluted environments. Biotechnol. Bioeng. 110, 220-228, doi:10.1002/Bit.24616 (2013).

18. Binder, S. et al. A high-throughput approach to identify genomic variants of bacterial metabolite producers at the single-cell level. Genome Biol. 13, doi:10.1186/Gb-2012-13-5-R40 (2012).

19. Mustafi, N., Grunberger, A., Kohlheyer, D., Bott, M. \& Frunzke, J. The development and application of a single-cell biosensor for the detection of L-methionine and branched-chain amino acids. Metab. Eng. 14, 449-457, doi:10.1016/j.ymben.2012.02.002 (2012).

20. Huber, R. et al. Robo-Lector - a novel platform for automated high-throughput cultivations in microtiter plates with high information content. Microb. Cell Fact. 8, doi:10.1186/1475-2859-8-42 (2009).

21. Keilhauer, C., Eggeling, L. \& Sahm, H. Isoleucine synthesis in Corynebacterium glutamicum: molecular analysis of the ilvB-ilvN-ilvC operon. J. Bacteriol. 175, 5595-5603 (1993).

22. Frunzke, J., Bramkamp, M., Schweitzer, J. E. \& Bott, M. Population Heterogeneity in Corynebacterium glutamicum ATCC 13032 caused by prophage CGP3. J. Bacteriol. 190, 5111-5119, doi:10.1128/JB.00310-08 (2008). 